\title{
TGase positively regulates photosynthesis via activation of Calvin cycle enzymes in tomato
}

\author{
Min Zhong ${ }^{1}$, Yu Wang ${ }^{1}$, Kun Hou', Sheng Shu ${ }^{1,2}$, Jin Sun ${ }^{1,2}$ and Shirong Guo ${ }^{1,2}$
}

\begin{abstract}
Transglutaminases (TGases), which are widespread cross-linking enzymes in plants, play key roles in photosynthesis and abiotic/biotic stress responses; however, evidence concerning the genetics underlying how TGase improves the capability of photosynthesis and the mechanism of TGase-mediated photosynthesis are not clear in this crop species. In this study, we clarified the function of TGase in the regulation of photosynthesis in tomato by comparing wild-type (WT) plants, tgase mutants generated by the CRISPR/Cas9 system and TGase-overexpressing (TGaseOE) plants. Our results showed that increasing the transcript level of TGase resulted in an enhanced net photosynthetic rate (Pn), whereas the tgase mutants presented significantly inhibited Pns and $\mathrm{CO}_{2}$ assimilation compared with the WT. Although the total RuBisCO activity was not affected by TGase, the initial and activation status of RuBisCO and the activity of RuBisCO activase (RCA) and fructose-1,6-bisphosphatase (FBPase) in TGaseOE plants were significantly higher than that in WT plants. Except for RuBisCO small subunit (RbcS), the transcription levels of Benson-Calvin cycle-related genes were positively related to the endogenous TGase activity. Furthermore, TGaseOE plants had higher protein levels of RuBisCO large subunit (RbcL) and RCA than did WT plants and showed a reduced redox status by enhancing the activity of dehydroascorbate reductase (DHAR) and glutathione reductase (GR), which was compromised in TGasedeficient plants. Overall, TGase positively regulated photosynthesis by maintaining the activation states of the Benson-Calvin cycle and inducing changes in cellular redox homeostasis in tomato.
\end{abstract}

\section{Introduction}

It is well known that photosynthesis is considered the most important metabolic process for biomass accumulation in plants but is limited by carbohydrates and various environmental cues. Therefore, understanding the mechanisms of regulation of photosynthetic processes and improving crop yield potential are critical. Interestingly, increasing evidence supports the hypothesis that polyamines (PAs) regulate photosynthetic capability ${ }^{1,2}$. It has been reported that PAs are usually found in the

\footnotetext{
Correspondence: Shirong Guo (srguo@njau.edu.cn)

${ }^{1}$ Key Laboratory of Southern Vegetable Crop Genetic Improvement, Ministry of Agriculture, College of Horticulture, Nanjing Agricultural University, 210095 Nanjing, China

${ }^{2}$ Suqian Academy of Protected Horticulture, Nanjing Agricultural University, 223800 Suqian, China
}

These authors contributed equally: Min Zhong, Yu Wang photosynthetic apparatus and are closely related to plant growth and stress responses ${ }^{3}$. Overexpressing the genes involved in PA biosynthesis results in increased photosynthetic rates (Pns) in transgenic plants ${ }^{4,5}$. In addition, putrescine (Put) plays a positive role in stimulating photophosphorylation, at least to some degree, and regulates adenosine triphosphate synthesis ${ }^{6,7}$. PAs are an important regulator of redox homeostasis, which increase photosynthesis because of the activation of antioxidant enzymes and the modulation of reactive oxygen species (ROS) homeostasis $^{8,9}$. However, the functions of PA-regulated photosynthesis are not fully understood.

The regulation of PAs is often mediated by transglutaminases (TGases), which are able to establish $\varepsilon$ - $(\gamma$-glutamyl) links with PAs for the posttranslational modification of proteins. For instance, PAs cross-linked with tubulin

\section{(c) The Author(s) 2019}

(c) (i) Open Access This article is licensed under a Creative Commons Attribution 4.0 International License, which permits use, sharing, adaptation, distribution and reproduction c. in any medium or format, as long as you give appropriate credit to the original author(s) and the source, provide a link to the Creative Commons license, and indicate if changes were made. The images or other third party material in this article are included in the article's Creative Commons license, unless indicated otherwise in a credit line to the material. If material is not included in the article's Creative Commons license and your intended use is not permitted by statutory regulation or exceeds the permitted use, you will need to obtain permission directly from the copyright holder. To view a copy of this license, visit http://creativecommons.org/licenses/by/4.0/. 
and actin via TGase have been observed during the germination of Malus domestica pollen ${ }^{10,11}$. TGases are intracellular and extracellular enzymes that are actively regulated by $\mathrm{Ca}^{2+}$ and can be negatively regulated by guanosine triphosphate. It has been demonstrated that TGases exhibit a large number of functions both in eukaryotes and prokaryotes ${ }^{12}$. In addition, a large amount of TGases are present in the mitochondria, cell walls, and chloroplasts of plants and are continually being identified $^{13-16}$. In addition, the first TGase gene discovered was AtPng1p, which is most studied gene encoding a TGase protein in Arabidopsis; AtPng1p is expressed under conditions of undisturbed growth and expressed ubiquitously during all stages of plant growth and can be induced by various light conditions ${ }^{14,17}$. Furthermore, TGases are delivered via a membrane/cytoskeleton-based transport system to the cell wall, where they regulate the apical growth of pollen tubes ${ }^{18}$. Numerous reports have shown that TGase plays roles not only in nonphotosynthetic tissues but also in photosynthetic organs. Recent proteomic and transcriptomic studies have shown that chloroplast proteins (i.e., light-harvesting complexes of photosystem II (LHCII) and chlorophyll-binding protein of 29,26 , and $24 \mathrm{kDa}$ ) are the target proteins of TGase in vivo ${ }^{19}$. Chloroplast-associated proteins such as the larger subunit of RuBisCO (RbcL) and photosystem II proteins are substrates of TGase in higher plants ${ }^{16,20}$. In this respect, TGase may have positive roles in photosynthesis or photoprotection reactions. Characterization of transgenic tobacco overexpressing TGase revealed that TGase has a positive role that involves the photosystems $^{21}$. In addition, photosynthetic complexes are often significantly affected by TGase ${ }^{22}$. However, the role of TGase in photosynthesis is still unclear. Researchers have been focused on the posttranslational modification of LHCII and the polyamination of thylakoids by TGase, but the function of TGase in redox regulation is often overlooked. PAs may be donors for the biosynthesis of amino acids such as glutamic acid, a key chlorophyll and glutathione precursor; in addition, PAs can regulate plastidial membrane assembly or take part in redox regulation, which has been indicated to be mediated by TGase $^{23}$. Moreover, some TGases have been identified in rice and maize, but their functions and regulatory mechanisms are largely unknown, especially in horticultural plant species.

Tomato is one of the most economically important horticultural crop species and is distributed worldwide, and this species been widely used to study photosynthesis. The clustered regulatory interspaced short palindromic repeats/CRISPR-associated protein 9 (CRISPR/Cas9) gene editing technology has been established and used to characterize gene functions in plants ${ }^{24}$. In this study, to analyze the potential role of TGase in tomato, we generated TGase-overexpressing (TGaseOE) plants and tgase mutants by CRISPR/Cas9 technology and compared their photosynthetic capability with that of wild-type (WT) plants. We found a positive relationship between endogenous TGase activity and the capability of photosynthesis. TGase improved photosynthetic capability by altering the cellular redox status and activating the antioxidant capability.

\section{Materials and methods \\ Plant materials and growth conditions}

Tomato (Solanum lycopersicum L. cv Ailsa Craig) was used for expression analysis. The seedlings were grown in a greenhouse at Nanjing Agriculture University in Nanjing city under standard water management and pest control regimens.

\section{Generation and selection of transgenic plants}

To generate TGaseOE plants, the CDS of TGase was obtained via PCR amplification using the primers $T G a$ seOE-F (5'-TTGGCGCGCCATGGTTGCTCGGAGACT CGCCGTTA-3') and TGaseOE-R (5'-CGGGGTACCACTGCTACCTGCAAAGAGGTCAATG-3') according to the sequence (Sol Genomic Network accession Solyc01g097440). The PCR product was inserted into the binary plasmid vector pFGC1008-HA with a 35S promoter after being digested with $A s c \mathrm{I}$ and $K p n \mathrm{I}$. The vector was then transformed into Agrobacterium tumefaciens strain EHA105, which was then inserted into Ailsa Craig plants following the method of Fillatti et $\mathrm{al}^{25}$. The overexpression of TGase in plants was identified by real-time quantitative PCR (qPCR) analysis (Fig. S1). Two homozygous F2 lines (OE-\#1 and OE-\#2) were chosen for the experiment.

To obtain mutants, we used the CRISPR/Cas9 genometargeting system to modify the TGase according to previous methods ${ }^{26}$. In the TGase coding region, we chose the guide RNA sequence GGCCCTTCAGTCTCATTACC as the single guide RNA (sgRNA). Doublestranded DNA, generated by annealing the oligo pairs, was inserted into an AtU6-sgRNA-AtUBQ-Cas9 vector, which was then digested by HindIII and KpnI and subsequently introduced into a pCAMBIA1301 vector using T4 DNA ligase (Thermo Fisher Scientific, Waltham, USA, EL0013). The integrated constructs were subsequently transformed into EHA105 bacteria, which were then inserted into Ailsa Craig plants; hygromycin was then used to screen the transgenic plants. The genomic DNA of the mutant seedlings was extracted for PCR using specific primers (Table S1). The PCR products of the mutants were detected by direct sequencing methods. The tgase- 1 and tgase- 2 mutants, which carried a $10 \mathrm{bp}$ deletion and a $1 \mathrm{bp}$ addition, respectively, were identified and used in this study (Fig. S2). 
In the experiments, five genotypes, Ailsa Craig (WT) plants, TGase-deficient mutants (tgase-1 and tgase-2), and overexpression plants (OE-\#1 and OE-\#2), were used. To analyze photosynthetic capability, germinated seeds were sown in growth media. After full development of the second true leaves, the seedlings were transplanted into $250 \mathrm{~cm}^{3}$ plastic pots that contained media and were watered with 1/2-strength Hoagland's nutrition solution. Forty-five-day-old plants were used for the experiment. For assay the Calvin cycle enzyme activity, leaf discs were harvested from different genotypes, then frozen immediately in liquid nitrogen, and stored at $-80^{\circ} \mathrm{C}$ prior to analysis. For detection the activity of TGase and antioxidant enzymes, the leaf samples were collected based on fresh weight. For analysis the gene expression, the whole leaflets were collected.

\section{TGase activity analysis}

The hydroxamate method was used to calculate the transglutaminase activity, with some modifications ${ }^{27}$. Leaf samples $(0.3 \mathrm{~g})$ were homogenized with phosphate buffer $(50 \mathrm{mM}, \mathrm{pH} 7.8)$ at $12,000 \mathrm{~g}$ for $15 \mathrm{~min}$ at $4{ }^{\circ} \mathrm{C}$ for endogenous TGase activity measurement. The colorimetric method $(450 \mathrm{~nm})$ was used to measure the activity of TGase in conjunction with $\mathrm{N}$-carbobenzoxy-l-glutami nylglycin (CBZ-Cln-Gly-OH), which is the specific substrate of TGase. The $100 \mu \mathrm{l}$ of the supernatant was fully mixed with the reaction $(50 \mathrm{mM}$ PBS, and $0.5 \mathrm{mM}$ CBZ-Cln-Gly-OH, pH 7.8), then performed at $30^{\circ} \mathrm{C}$ for $10 \mathrm{~min}$ and performed with $5 \mathrm{mM} \mathrm{Ca}{ }^{2+}$; replaced by $1 \mathrm{mM}$ EDTA in the negative control. L-glutamic acid $\gamma$ mono-hydroxamate was used to prepare the standard curve. The enzyme activity was defined as the amount of enzyme that catalyzed the formation of $1 \mu \mathrm{mol}$ of hydroxamate/min.

\section{Leaf gas exchange measurements}

Forty-five-day-old plants were used for comparing photosynthetic capabilities. Gas exchange analysis was performed via an LI-6400 portable photosynthesis system (LI-6400; LI-COR, Lincoln, NE, USA). An assimilation vs intercellular $\mathrm{CO}_{2}$ concentration $(\mathrm{A} / \mathrm{Ci})$ curve was generated as described by Caemmerer and Farquhar ${ }^{28}$. The maximum rates of Rubisco $\left(V_{\mathrm{c}, \max }\right)$ and the maximum rate of electron transport for RuBP regeneration $\left(J_{\max }\right)$ were measured according to the $\mathrm{A} / \mathrm{Ci}$ curves as described by Ethier and Livingston ${ }^{29}$.

\section{Determination of Rubisco, Rubisco activase (RCA), and fructose-1, 6-bisphosphatase (FBPase) activity}

The Rubisco activity was measured following the method of Ward and Keys ${ }^{30}$, with slight modifications. Leaf samples were ground with extraction buffer (2\% insoluble PVPP, $1 \mathrm{mM}$ EDTA, $50 \mathrm{mM}$ HEPES, $10 \mathrm{mM}$
$\mathrm{MgCl}_{2}$, and $10 \mathrm{mM} \beta$-mercaptoethanol; $\left.\mathrm{pH} 8.0\right)$ and then centrifuged at $12,000 \mathrm{~g}$ for $15 \mathrm{~min}$ at $4{ }^{\circ} \mathrm{C}$. The crude extract was used to assay the total activity. Activation was performed in a $100 \mu \mathrm{l}$ mixture solution at $28^{\circ} \mathrm{C}$ for $15 \mathrm{~min}$. Initial Rubisco activity was determined in a $100 \mu \mathrm{l}$ reaction medium, and the change in absorbance within $90 \mathrm{~s}$ was measured at $340 \mathrm{~nm}$. The RCA activity was measured with a Rubisco Activase Assay Kit (Genmed Scientifics, Wilmington, USA) according to the manufacturer's instructions.

The activity of FBPase was detected by measuring the increase at $A_{340 \mathrm{~nm}}$ as previously described ${ }^{31}$. The total activity of FBPase was determined using the crude extract, which was activated in a $100 \mu \mathrm{l}$ activation mixture reaction (0.1 M Tris- $\mathrm{HCl}$ ( $\mathrm{pH}$ 8.0), $2 \mathrm{mM}$ fructose-1,6bisphosphate (FBP), $100 \mathrm{mM}$ dithiothreitol (DTT), and $10 \mathrm{mM} \mathrm{MgCl} 2$ ). After homogenization, the initial activity was measured immediately. The reaction was started by the addition of the enzyme extract.

\section{Glutathione content and ascorbate content assays}

To determine the glutathione and ascorbate contents, leaf tissue was ground in $2 \mathrm{ml}$ of extraction buffer ( $6 \%$ metaphosphoric acid) and then centrifuged at $12,000 \mathrm{~g}$ for $10 \mathrm{~min}$ at $4{ }^{\circ} \mathrm{C}$, after which the supernatant was used for further analysis. To assay the GSH and oxidized glutathione (GSSG), the neutralized supernatant with phosphate buffer $(0.5 \mathrm{M}, \mathrm{pH} 7.5)$ was added to the reaction mixture (100 mM phosphate buffer, $5 \mathrm{mM}$ EDTA, $0.2 \mathrm{mM}$ $\mathrm{NADPH}$, and $0.6 \mathrm{mM}$ 5,5'-dithio-bis (2-nitrobenzoic acid), $\mathrm{pH}$ 7.5). After adding the glutathione reductase (GR) (Sigma, Santa Clara, CA, USA) to start the reaction, the changes in absorbance within $1 \mathrm{~min}$ at $412 \mathrm{~nm}$ were measured. The GSH was masked after adding $40 \mu \mathrm{l}$ of 2vinylpyridine to the supernatant for the GSSG assay and the total glutathione assay by adding $40 \mu \mathrm{l}$ of water. The GSH content was measured by removing the GSSG content from the total content ${ }^{32}$.

To assay the total levels of AsA, $20 \mu$ of the supernatant was transferred to a new tube containing potassium phosphate buffer $(0.5 \mathrm{M}, \mathrm{pH} 7.4)$; the same amount of $5 \mathrm{mM}$ DTT was added as was the supernatant, after which the mixture was incubated at $37^{\circ} \mathrm{C}$ for $20 \mathrm{~min}$. Afterward, $10 \mu \mathrm{l}$ of $\mathrm{N}$-ethylmaleimide (NEM, $0.5 \% \mathrm{w} / \mathrm{v}$ ) was added to clear the excess DTT via incubation for $1 \mathrm{~min}$ at $25^{\circ} \mathrm{C}$. A color reagent $(80 \mu \mathrm{l})$ (see below) was added to the mixture, which was then incubated at $37^{\circ} \mathrm{C}$ for $1 \mathrm{~h}$; the absorbance was measured at $550 \mathrm{~nm}$. To analyze the reduced AsA, $20 \mu \mathrm{l}$ of potassium phosphate (0.4 M, pH 7.4) was replaced with DTT and NEM, and the procedure was performed according to the total AsA assay. The color reagent was as follows: solution A contained 31\% orthophosphoric acid, $0.6 \%(\mathrm{w} / \mathrm{v})$ iron chloride $\left(\mathrm{FeCl}_{3}\right)$, and $4.6 \%(\mathrm{w} / \mathrm{v}) \mathrm{TCA}$, whereas solution B contained 4\% 2,2-dipyridyl. 


\section{Measurements of the activity related to AsA-GSH cycle enzymes}

To analyze the enzymatic activities related to the AsA-GSH cycle, leaf samples were ground in $3 \mathrm{ml}$ of extraction buffer (25 mM HEPES, 2\% PVP, $2 \mathrm{mM}$ ascorbic acid, and $0.2 \mathrm{mM}$ EDTA, $\mathrm{pH} 7.8$ ) and centrifuged at $12,000 \mathrm{~g}$ for $20 \mathrm{~min}$ at $4{ }^{\circ} \mathrm{C}$. The supernatants were then collected and used for the enzymatic activity assay. The activities of ascorbate peroxidase (APX) and dehydroascorbate reductase (DHAR) were evaluated by monitoring the decrease and increase in absorbance at $290 \mathrm{~nm}$ and $265 \mathrm{~nm}$, respectively, as described by Nakano and Asada $^{33}$. To analyze the activity of GR, we monitored the rate of NADPH decrease according to the method reported by Halliwell and Foyer ${ }^{34}$.

\section{RNA isolation and reverse-transcription qPCR}

Tomato leaves were used to extract total RNA following the manufacturer's instructions of an RNA extraction kit (Tiangen, Beijing, China). Total RNA $(1 \mu \mathrm{g})$ was used to synthesize cDNA using a HiScript ${ }^{\mathrm{m}} \mathrm{Q}$ RT SuperMix for qPCR (+gDNA wiper) kit (Vazyme, Nanjing, China). qPCR analyses were performed using an ABI ViiA7 realtime PCR system (Applied Biosystems, CA, USA). The PCR program consisted of predenaturation at $95^{\circ} \mathrm{C}$ for $5 \mathrm{~min}$, followed by 40 cycles of $95^{\circ} \mathrm{C}$ for $10 \mathrm{~s}$ and $60{ }^{\circ} \mathrm{C}$ for $30 \mathrm{~s}$. The Actin gene was used as a reference gene, and the qPCR-specific primers are presented in supplementary Table S1. The relative levels for each expression were calculated according to the methods of Livak and Schmittgen $^{35}$.

\section{Western blot analysis}

Proteins were extracted as described previously ${ }^{35}$. The proteins were resolved using $12 \%(\mathrm{w} / \mathrm{v})$ SDS-polyacrylamide gel electrophoresis and then transferred to a nitrocellulose membrane (Millipore, Saint-Quentin, France) ${ }^{36}$. RuBisCO large subunit ( $\mathrm{RbcL}), \mathrm{RuBisCO}$ small subunit (RbcS), and RCA were detected with commercial antibodies (Agrisera, Vannes, Sweden). At least three independent replicates were used for each determination. The accumulation of proteins was quantified using Quantity One software (Bio-rad, Hercules, California, CA, USA).

\section{Statistics}

The data are presented as the means \pm SDs and were analyzed by SPSS 20 statistical software. The experimental data were analyzed with Duncan's multiple range test at $P$ $<0.05$.

\section{Results}

\section{Expression pattern of TGase in tomato}

To detect the expression pattern of TGase, the mRNA transcripts of TGase were measured in different tissues, including the roots, stems, leaves, flowers, and fruits, in tomato (Fig. S1). Transcripts of TGase were highly expressed in the leaves and fruits and accumulated in the flowers (Fig. S1).

To explore the functions of TGase, we first generated TGaseOE tomato lines and obtained several independent transgenic lines. Moreover, the transcripts of TGase in two overexpression lines, OE-\#1 and OE-\#2, showed 117.5 and 124.3 times greater expression than the WT plants did, respectively (Fig. S2). We also used the CRISPR/Cas9 system to generate mutants, which were named tgase- 1 and tgase-2, with a loss of 10 bp and an "A" insertion in an exon of TGase, respectively (Fig. S3). In the mutants, the activity of TGase was almost undetectable; in contrast, the activity of TGase increased in the TGaseOE plants (Fig. S4). These results indicated that TGase deficiency significantly inhibited TGase activity but dramatically increased TGaseOE activity. These homozygous overexpressing lines and mutants were used in subsequent experiments.

\section{TGase enhanced $\mathrm{CO}_{2}$ assimilation in tomato plants}

TGases are closely related to photosynthesis in plants. To reveal the role of TGase in photosynthesis capability, we first analyzed the differences in the parameters of gas exchange among the WT, tgase-1, tgase-2, OE-\#1, and OE-\#2 plants. The results indicated that deficiency of TGase significantly inhibited net Pns compared to WT (Fig. 1a). Moreover, other gas exchange parameters, such as stomatal conductance (Gs), $\mathrm{C}_{\mathrm{i}}$, and transcription rate $(\mathrm{Tr})$, were also reduced in the tgase mutants (Fig. $1 \mathrm{~b}-\mathrm{d}$ ). In contrast, the Pn, Gs, $\mathrm{Ci}$, and $\mathrm{Tr}$ increased in the OE-\#1 and OE-\#2 lines compared with WT plants (Fig. 1a-d). To further examine the possible mechanism of TGase regulation of photosynthesis, we used Farquhar's model to analyze the $V_{\mathrm{c}, \max }$ and the $J_{\max }$. The results showed that TGase deficiency decreased $V_{\mathrm{c}, \max }$ and $J_{\max }$; however, compared with those in WT, $V_{\mathrm{c}, \max }$, and $J_{\max }$ increased in the TGaseOE plants (Fig. 1e, f). Thus, TGase-mediated $\mathrm{CO}_{2}$ assimilation and RuBP carboxylation and regeneration capabilities in tomato plants.

\section{TGase promoted the gene expression and activity of the Benson-Calvin cycle}

To understand the possible role of TGase in regulating the capability of photosynthesis, we further analyzed the activity of FBPase, which plays key roles in RuBP regeneration. The results showed that changes in the endogenous TGase activity did not induce a significant change in total Rubisco activity (Fig. 2a), but the initial Rubisco activity showed significantly differed among the different genotypes, resulting in a decreased and increased Rubisco activation state in the tgase mutants and TGaseOE plants, respectively (Fig. 2b, c). Similarly, the activity of RCA was 


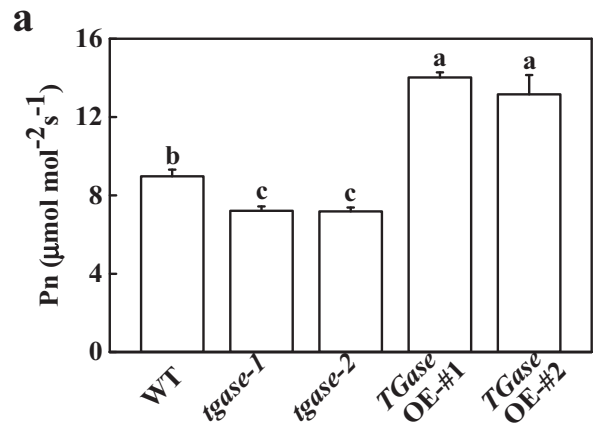

c

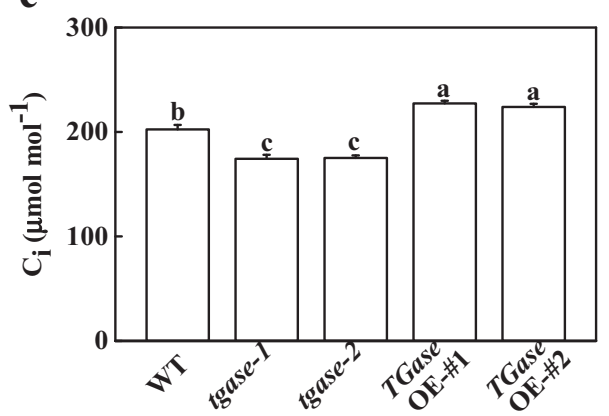

e

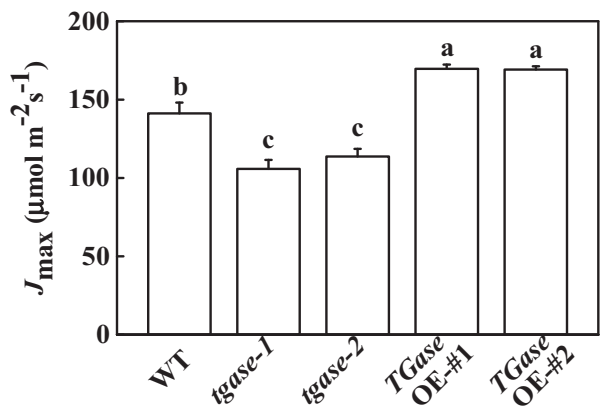

b

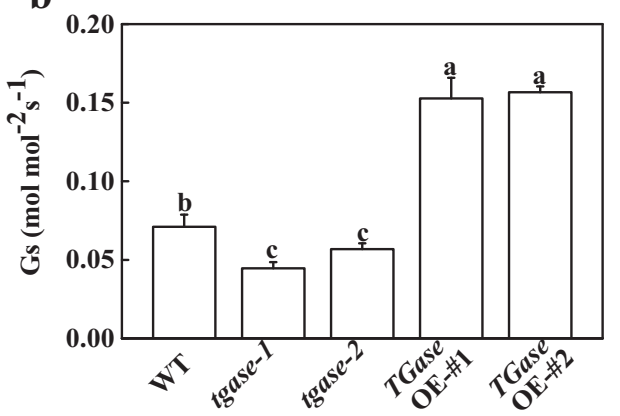

d

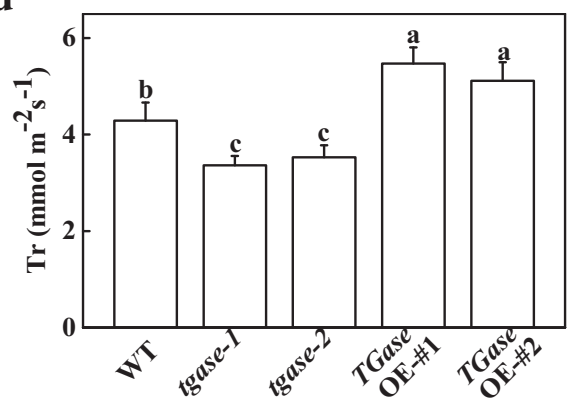

f

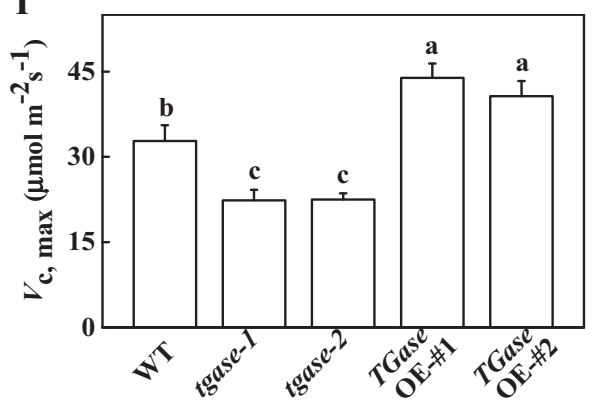

Fig. 1 Effects of TGase overexpression (OE-\#1 and OE-\#2) and mutation (tgase-1 and tgase-2) on photosynthesis. Net photosynthetic rate (Pn) (a), stomatal conductance $(\mathrm{Gs})(\mathbf{b})$, intercellular $\mathrm{CO}_{2}$ concentration (Ci) (c), transpiration rate ( $\mathrm{Tr}$ ) (d), maximum ribulose-1,5-bisphosphate (RuBP) regeneration rate $\left(J_{\max }\right)(\mathbf{e})$, and maximum ribulose-1,5-bisphosphate carboxylase/oxygenase (Rubisco) carboxylation rate $\left(V_{c, \text { max }}\right)(\mathbf{f})$. Each histogram represents a mean \pm SE of three independent experiments $(n=3)$. Different letters indicate significant differences between treatments $(P<0.05)$ according to Duncan's multiple range test

reduced in tgase mutants but elevated in TGaseOE plants (Fig. 2d). Moreover, tgase-1 and tgase-2 presented decreased the total FBPase activity, while high FBPase activity was detected in the TGaseOE plants (Fig. 2e). Furthermore, there was a similar change in the initial FBPase activity in the TGaseOE plant (Fig. 2f).

To further analyze the function of TGase in the regulation of photosynthesis, we examined the transcripts of genes related to the Benson-Calvin cycle, including $R C A$, $R b c L, R b c S$, glyceraldehyde-3-phosphate dehydrogenase, FBPase, sedoheptulose-1,7-bis-phosphatase, ribulose-5- phosphate kinase, and glycerate-3-phosphate kinase. Except for the expression level of the $r b c S$ gene, the expression levels of the other genes were upregulated in TGaseOE plants (Fig. 3). In contrast, deficiency of the TGase gene led to downregulation of these genes (Fig. 3). Moreover, the protein levels of rbcL, rbcs, and RCA presented similar results as those of their transcription levels. The results showed that TGase deficiency and overexpression induced decreases and increases in $\mathrm{rbcL}$ and RCA contents, respectively (Figs. 4 and S5), which are consistent with the total Rubisco activity. 

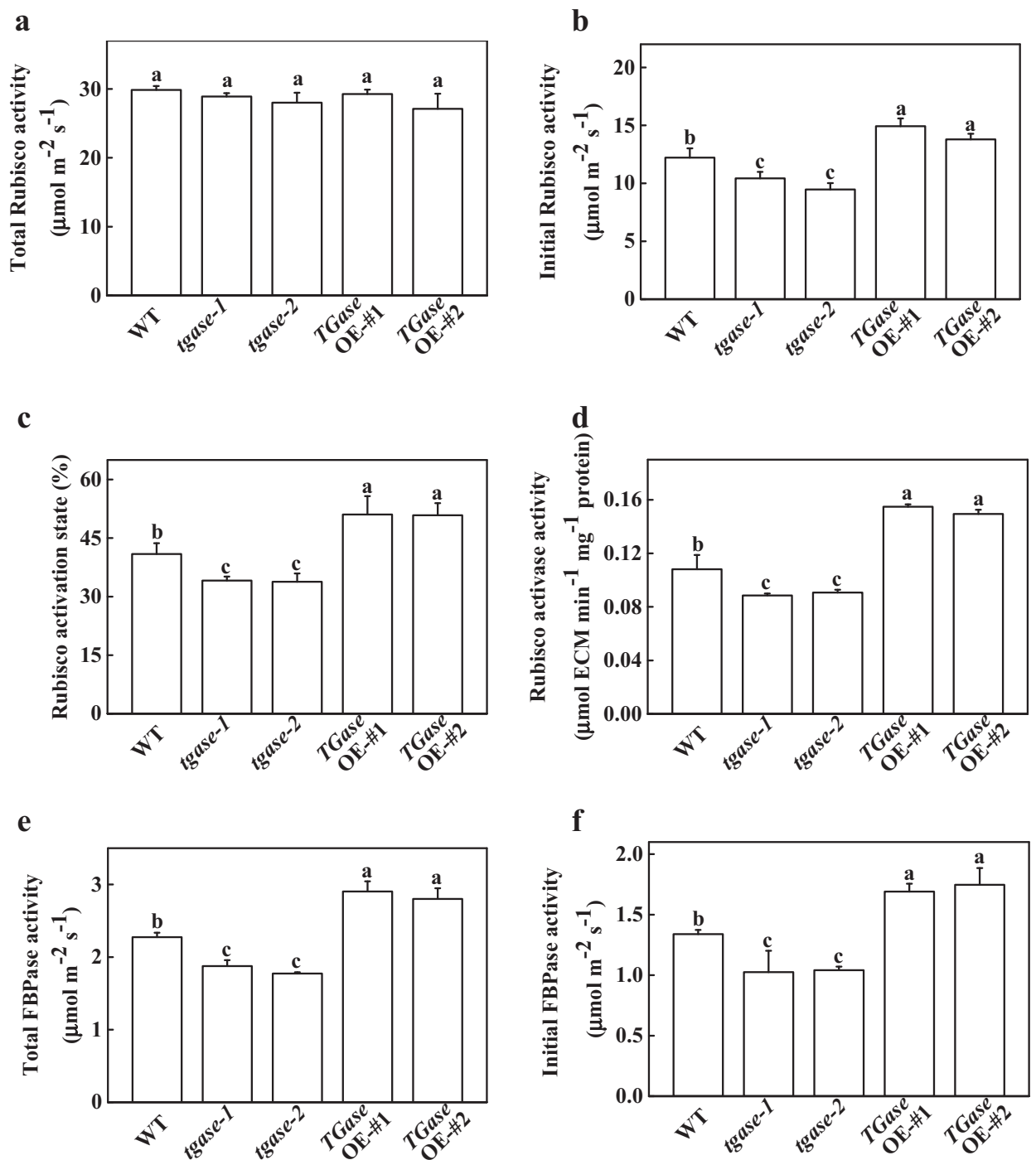

Fig. 2 Effects of TGase gene overexpression (OE-\#1 and OE-\#2) and mutation (tgase-1 and tgase-2) on the total and initial carboxylation activity of Rubisco $(\mathbf{a}, \mathbf{b})$, the activation status of Rubisco $(\mathbf{c})$, the activity of Rubisco activase (d), and total and initial activity of fructose-1,6-bisphosphatase (FBPase) (e, f). Each histogram represents a mean \pm SE of three independent experiments $(n=3)$. Different letters indicate significant differences between treatments $(P<0.05)$ according to Duncan's multiple range test

\section{TGase induced a reduced redox status and activated antioxidant enzymes}

It is well known that redox posttranslational modifications can regulate Calvin cycle enzymes. To reveal the mechanism by which TGase mediates Rubisco and RCA activity, we further examined the different status of glutathione and ascorbate contents. The GSH content was decreased in tgase mutants, whereas high TGase activity in the TGaseOE plants resulted in GSH content accumulation compared to WT (Fig. 5a). However, TGase deficiency led to an increase in GSSG content but a decrease in the TGaseOE plants compared to WT (Fig. 5a). The changes in total glutathione (GSH + GSSG) were similar to those of GSH (Fig. 5a). Importantly, the ratio of
GSH/GSSG showed a decline in the tgase mutants, whereas it increased in the TGaseOE plants (Fig. 5a). The content of AsA was not affected by different TGase activity levels in plants (Fig. 5b). TGase deficiency led to an increase in the oxidized ascorbate (DHA) content but a significant decrease in AsA in the TGaseOE lines (Fig. 5b). Although the total ascorbate (AsA + DHA) content was not influenced by the modulation of TGase activity, the AsA/DHA ratio significantly decreased and increased in the tgase mutants and overexpression plants, respectively (Fig. 5b).

TGase can maintain cellular redox status by mediating the enzymatic activity of the AsA-GSH cycle. In this study, APX, DHAR, and GR, which are involved in the 

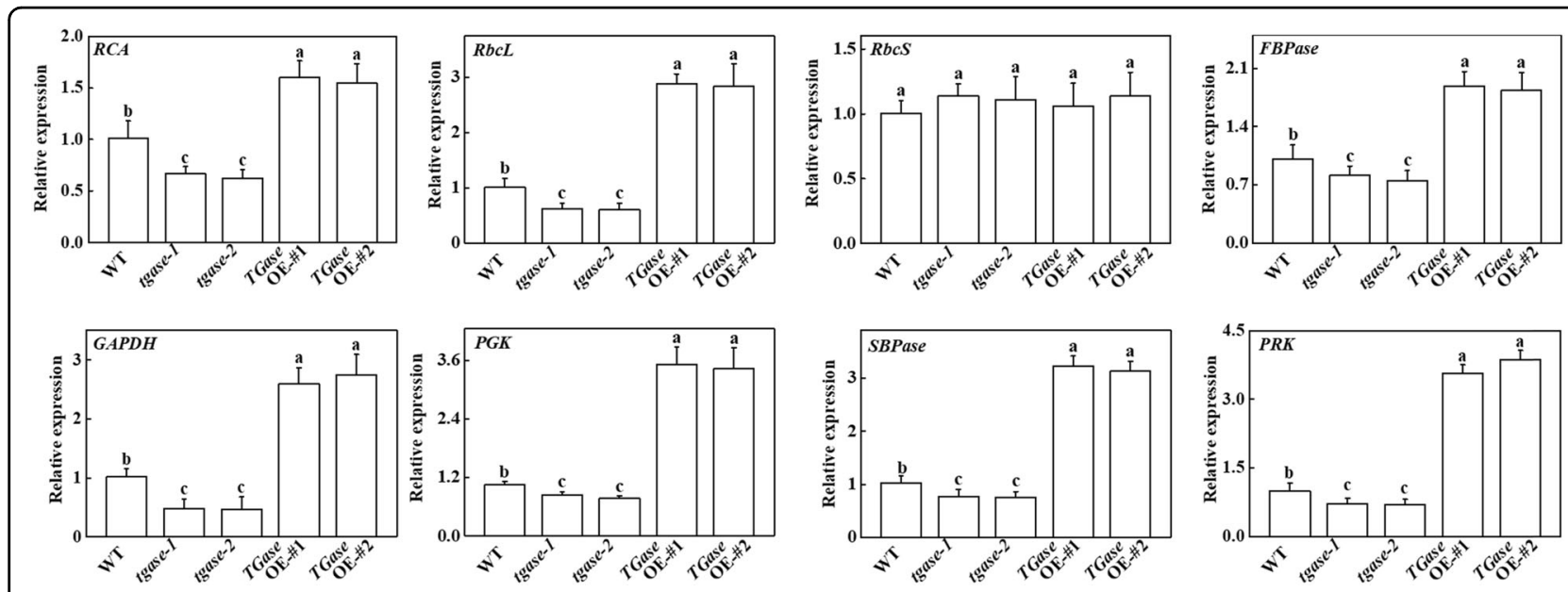

Fig. 3 Changes in the expression of Benson-Calvin cycle-related genes in overexpression (OE-\#1 and OE-\#2) and mutant (tgase-1 and tgase-2) plants. Each histogram represents a mean \pm SE of three independent experiments $(n=3)$. Different letters indicate significant differences between treatments $(P<0.05)$ according to Duncan's multiple range test

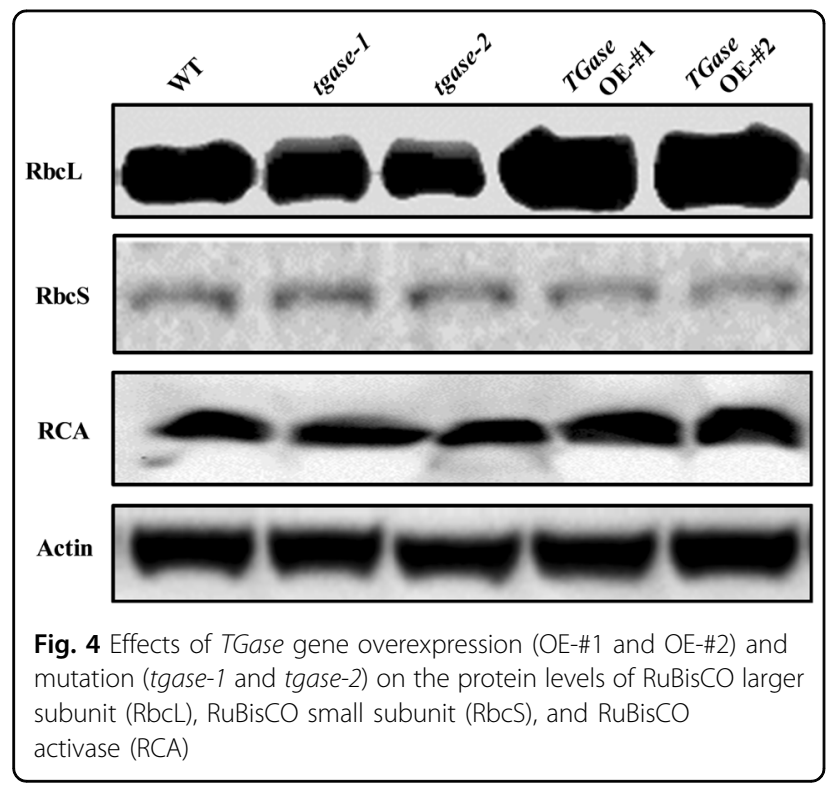

AsA-GSH cycle, were analyzed. The results showed that those activities were inhibited in the tgase mutants but were induced in the TGaseOE plants (Fig. 6a-c). Moreover, the expression of $G R$ was consistent with its activity (Fig. 6c, d).

\section{Discussion}

TGases are widely distributed in plants and have been found to be related to all stages of plant growth, programmed cell death, senescence, and stress ${ }^{37}$. However, until now, there has been no suitable transgenic plant with decreased TGase activity. Moreover, the best tobacco and Arabidopsis experimental lines overexpressed the plastidial transglutaminase from maize, but researchers have not analyzed whether the photosynthetic capability changes in the overexpression plants resulted from the alteration of the Calvin cycle and the promotion of carbohydrate metabolism ${ }^{21,38,39}$. In this study, we explored the function of TGase using a reverse genetic strategy. We found that high endogenous TGase activity is involved in the enhancement of photosynthesis in TGaseOE plants, but TGase deficiency led to decreased photosynthetic capability (Fig. 1), which suggested that TGase may play crucial roles in the regulation of photosynthesis. The reduction in the rate of photosynthesis in the tgase mutants was associated with a decrease in Gs and Ci (Fig. 1). Our previous study suggested that the application of $8 \mathrm{mM}$ Put enhances stomatal opening and promotes photosynthesis in cucumber ${ }^{40}$. Here, we also observed increased $\mathrm{Ci}$ in the TGase OE plants but not in the mutants. These results might be due to the positive effect of TGase on the PA level change, which resulted in a significant positive correlation between PAs and the Pn.

To date, several studies have reported that TGase regulates the thylakoid structure and $\mathrm{LHCII}^{22,41}$. For example, overexpression of the maize TGase could remodel tobacco thylakoids ${ }^{38,42}$. However, little is known about whether TGase can regulate the Calvin cycle and its associated processes. Hence, in this study, we used the A/ $\mathrm{Ci}$ curve and the Rubisco and FBPase activities to show some evidence for TGase mediation of photosynthetic capability due to the positive regulation of Calvin cycle enzyme activity. We observed that Calvin cycle-related genes were downregulated in the tgase mutant (Fig. 3). Furthermore, TGase deficiency also led to a significant decline in RCA activity and initial Rubisco and FBPase activity, as well as a reduction in $V_{c, \max }$ and activity of RCA, with almost no change in the total activity of 


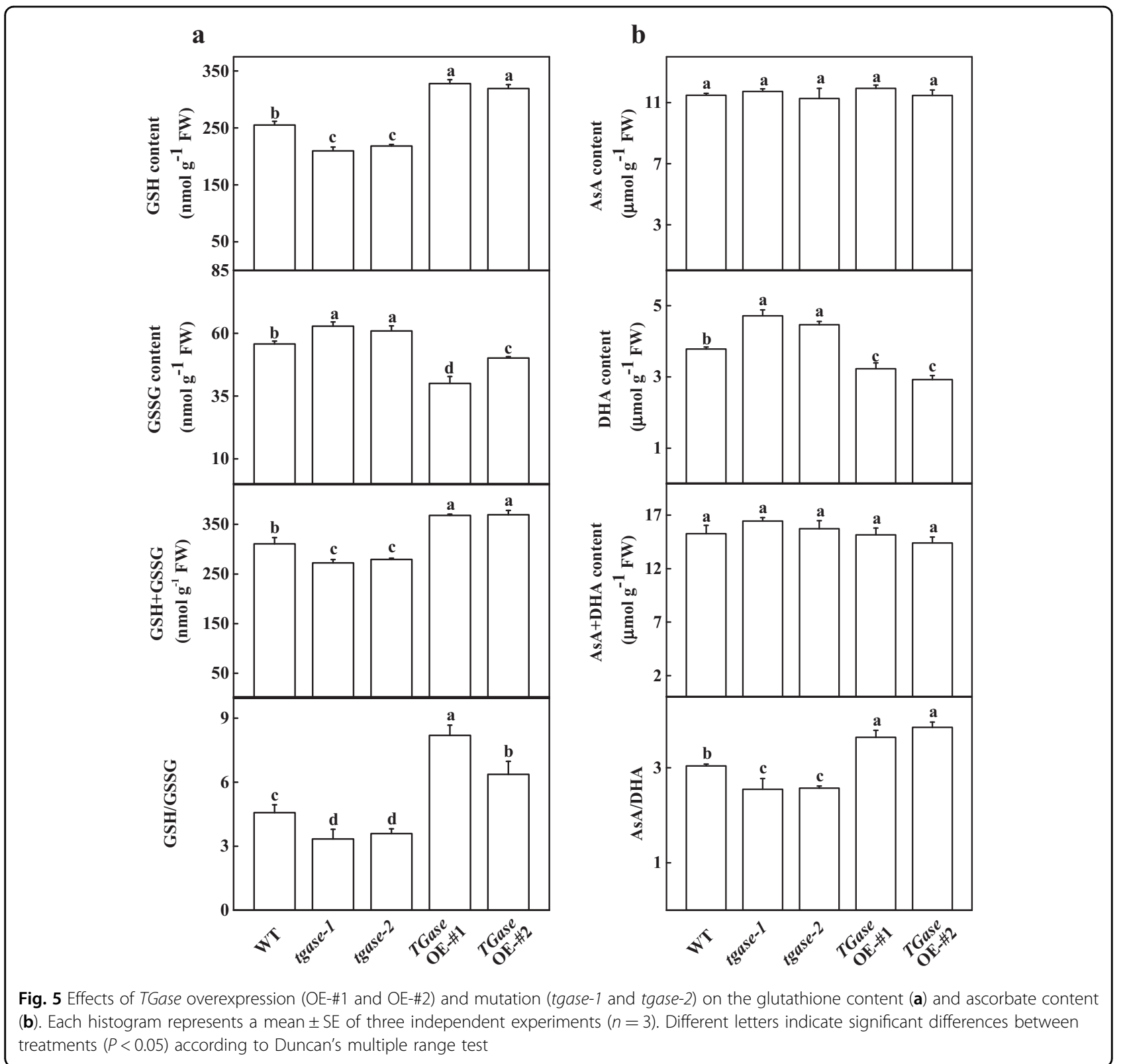

Rubisco (Figs. 2 and 4). In contrast, overexpression of TGase increased the expression and activity of these genes (Figs. 2 and 3), indicating that TGase mainly regulated the action of Rubisco activities to activate the state of Rubisco. $r b c S$ and $r b c L$ encode small and large Rubisco subunits, respectively, which compose the Rubisco holoenzyme and are tightly coordinated, but the transcripts of $r b c S$ and $r b c L$ are not correlated ${ }^{43}$. Indeed, although TGase positively mediates the transcription of $r b c L$, it did not mediate the expression level of $r b c S$ (Fig. 3b, c). This phenomenon may be due to the synthesis of rbcS being different because a study showed that overexpression of $r b c S$ did not lead to an increase in the content of Rubisco $^{44}$. Alternatively, pools of cellular glutathione could be a repressor of rbcL proteins when glutathione is in the oxidized state ${ }^{45}$. Considering that the redox status of glutathione was regulated by TGase, it is possible that TGase affects the levels of $r b c L$ (Fig. 5). The regeneration of RuBP is involved in not only the electron transport chain of photosynthesis but also the Rubisco enzyme in the Calvin cycle ${ }^{46}$. Both the expression and initial activity of FBPase were reduced in the tgase mutant plants (Fig. 2f). The lack of activation of RCA or FBPase was often related to a decline in $J_{\max }$ (Fig. 1e), and cellular glutathione homeostasis is related to the activities of these enzyme ${ }^{47}$.

Some reports have shown that GSH plays a key role in Rubisco activation by enhancing thiol and disulfide 


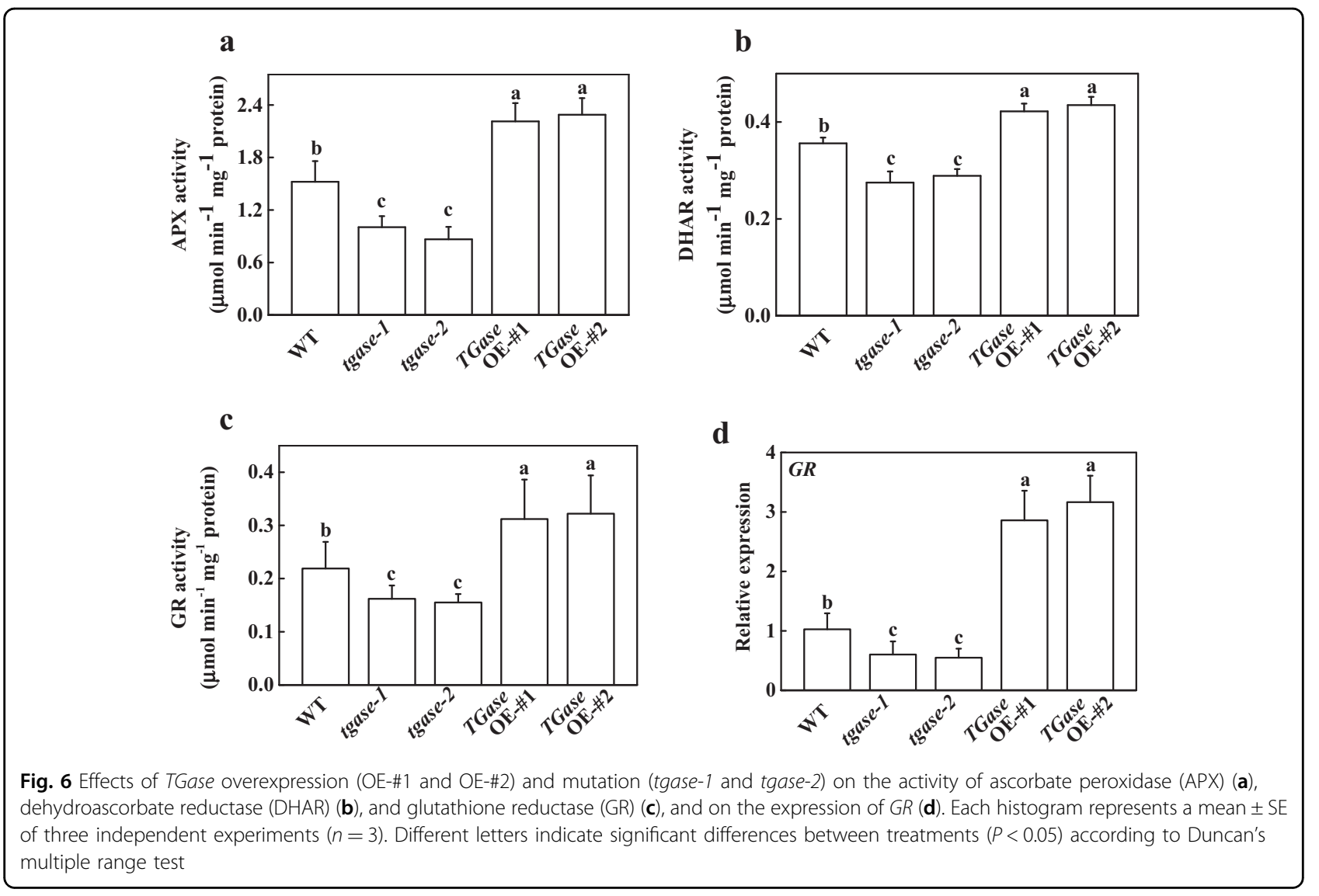

exchanges $^{48}$. The high ratio of GSH to GSSG increased the activation level of RuBisCO and consequently $\mathrm{CO}_{2}$ assimilation ${ }^{49}$. In addition, the glutathione redox could regulate translation of RbcL through modulating the ROS in the chloroplast $^{50}$. Overexpression of S-adenosylmethionine synthetase 1 (a PA biosynthesis gene) significantly increased the AsA/DHA and GSH/GSSG ratios in tomato $^{48,51,52}$. The mechanism of TGase positively regulates the activation state of Rubisco, which may be involved in glutathione redox status changes. Interestingly, in the present study, high ratios of GSH/GSSG and AsA/DHA were observed in TGaseOE lines (Fig. 5), which was caused by an increase in MDAR and GR enzymatic activity (Fig. 6). Activation of the GSH-AsA cycle in TGaseOE plants could maintain a reducing state of the chloroplast, which helps to activate Rubisco. In addition, TGase could bind to the large subunit of ribulose bisphosphate carboxylase-oxygenase, thereby directly activating the activity of Rubisco ${ }^{16}$. This phenomenon is in agreement with the results by which high TGase activity promoted RCA activity and protein content in TGaseOE plants (Figs. 2d and 4). On the other hand, maintaining the high AsA/DHA and GSH/GSSG ratios may be involved in hormone signaling, cell division, and other physiological processes ${ }^{49}$.
It is well known that maintaining cellular redox status requires a turnover rate of the ascorbate-glutathione cycle, and APX activity may have an important role in ROS scavenging ${ }^{53}$. Here, we found that a deficiency in TGase resulted in a decreased activity of antioxidantrelated enzymes, while overexpression of TGase increased their activities, as observed in the overexpression of maize plastidial TGase in tobacco ${ }^{42}$. These results indicated that high endogenous TGase activity induced antioxidant enzymes to regulate the redox system of the plants. This finding further revealed the cross-link between TGase and the glutathione system ${ }^{42}$.

In conclusion, the current study indicated that manipulation of endogenous TGase activity by overexpression of TGase could promote the $\mathrm{CO}_{2}$ assimilation rate through activating the Calvin cycle enzymes. Moreover, TGase-inducible changes in cellular redox homeostasis may be involved in activation of Calvin cycle enzymes.

\section{Acknowledgements}

We are grateful to Prof. Jie Zhou of Zhejiang University for the tomato seeds and transformation vectors. This work was supported by the National Natural Science Foundation of China (31672199 and 31801902), the China Earmarked Fund for Modern Agro-industry Technology Research System (CARS-23-B12) 
and the Fundamental Research Funds for the Central Universities (KJQN201928)

\section{Author contributions}

S.G. designed the experiment. M.Z. and Y.W. performed the experiments and wrote the manuscript; K.H. collected the plant materials and prepared the figures; and S.S. and J.S. analyzed the data and modified the manuscript. All authors reviewed and approved the manuscript.

\section{Conflict of interest}

The authors declare that they have no conflict of interest.

Supplementary Information accompanies this paper at (https://doi.org/ 10.1038/s41438-019-0173-z).

Received: 20 February 2019 Revised: 21 May 2019 Accepted: 6 June 2019 Published online: 01 August 2019

\section{References}

1. Shu, S. et al. The role of putrescine in the regulation of proteins and fatty acids of thylakoid membranes under salt stress. Sci. Rep. 5, 14390 (2015).

2. Velikova, V., Yordanov, I. \& Edreva, A. Oxidative stress and some antioxidant systems in acid rain-treated bean plants: protective role of exogenous polyamines. Plant Sci. 151, 59-66 (2000).

3. Hamdani, S., Yaakoubi, H. \& Carpentier, R. Polyamines interaction with thylakoid proteins during stress. J. Photochem. Photobiol. B. 104, 314-319 (2011).

4. Wi, S. J., Kim, W. T. \& Park, K. Y. Overexpression of carnation Sadenosylmethionine decarboxylase gene generates a broad-spectrum tolerance to abiotic stresses in transgenic tobacco plants. Plant Cell Rep. 25, 1111-1121 (2006)

5. Qi, Y. C., Wang, F. F., Zhang, H. \& Liu, W. Q. Overexpression of suadea salsa Sadenosylmethionine synthetase gene promotes salt tolerance in transgenic tobacco. Acta Physiol. Plant. 32, 263-269 (2010).

6. Ioannidis, N. E. \& Kotzabasis, K. Effects of polyamines on the functionality of photosynthetic membrane in vivo and in vitro. Biochim. Biophys. Acta 1767, 1372-1382 (2007).

7. Ioannidis, N. E., Sfichi, L. \& Kotzabasis, K. Putrescine stimulates chemiosmotic ATP synthesis. Biochim. Biophys. Acta 1757, 821-828 (2006).

8. Gupta, K., Dey, A. \& Gupta, B. Plant polyamines in abiotic stress responses. Acta Physiol. Plant. 35, 2015-2036 (2013).

9. Saha, J. et al. Polyamines as redox homeostasis regulators during salt stress in plants. Front. Environ. Sci. 3, 21 (2015).

10. Serafini-Fracassini, D. \& Stefano, D. D.Transglutaminases: widespread crosslinking enzymes in plants. Ann. Bot. 102, 145-152 (2008).

11. Cai, G., Serafini-Fracassini, D. \& Del Duca, S. Regulation of pollen tube growth by transglutaminase. Plants 2, 87-106 (2013).

12. Lorand, L. \& Graham, R. M. Transglutaminases: crosslinking enzymes with pleiotropic functions. Nat. Rev. Mol. Cell Boil. 4, 140 (2003).

13. Lilley, G. R., Skill, J., Griffin, M. \& Bonner, P. L. Detection of $\mathrm{Ca}^{2+}$-dependent transglutaminase activity in root and leaf tissue of monocotyledonous and dicotyledonous plants. Plant Physiol. 117, 1115-1123 (1998).

14. Della Mea, M., Caparrós-Ruiz, D., Claparols, I., Serafini-Fracassini, D. \& Rigau, JAtPng1p first plant transglutaminase. Plant Physiol. 135, 2046-2054 (2004).

15. Della, M. M., De, F. F., Genovesi, V., Serafini, F. D. \& Del, D. S. The acropetal wave of developmental cell death of tobacco corolla is preceded by activation of transglutaminase in different cell compartments. Plant Physiol. 144, 1211-1222 (2007).

16. Margosiak, S. A. et al. Identification of the large subunit of ribulose 1,5bisphosphate carboxylase/oxygenase as a substrate for transglutaminase in Medicago sativa L. (Alfalfa). Plant Physiol. 92, 88-96 (1990).

17. Andreas, D., Guangtao, L., Lennarz, W. J., Thorsten, N. \& Frédéric, B. The Arabidopsis AtPNG1 gene encodes a peptide: N-glycanase. Plant J. 52, 94-104 (2010).

18. Del Duca, S. et al. Distribution of transglutaminase in pear pollen tubes in relation to cytoskeleton and membrane dynamics. Plant Physiol. 161, 1706-1721 (2013).
19. Campos, N. et al. Proteomic and transcriptomic analysis of rice tranglutaminase and chloroplast-related proteins. Plant Sci. 229, 142-153 (2014).

20. Dondini, L. et al. Suborganellar localisation and effect of light on Helianthus tuberosus chloroplast transglutaminases and their substrates. Planta 217, 84-95 (2003).

21. Zhong, $M$. et al. Overexpression of transglutaminase from cucumber in tobacco increases salt tolerance through regulation of photosynthesis. Int. J. Mol. Sci. 20, 894 (2019).

22. Della, Mea et al. A Zea mays 39-kDa thylakoid transglutaminase catalyses the modification by polyamines of light-harvesting complex $\|$ in a lightdependent way. Planta 219, 754-764 (2004).

23. Sobieszczuk-Nowicka, E. \& Legocka, J. Plastid-associated polyamines: their role in differentiation, structure, functioning, stress response and senescence. Plant Biol. 16, 297-305 (2014).

24. Liu, H. et al. CRISPR-P 2.0: an improved CRISPR-Cas9 tool for genome editing in plants. Mol. Plant. 10, 530-532 (2017).

25. Fillatti, J. J., Kiser, J., Rose, R. \& Comai, L. Efficient transfer of a glyphosate tolerance gene into tomato using a binary Agrobacterium tumefaciens vector. Nat. Biotechnol. 5, 726 (1987)

26. Pan, C. et al. CRISPR/Cas9-mediated efficient and heritable targeted mutagenesis in tomato plants in the first and later generations. Sci. Rep. 6, 24765 (2016).

27. Grossowicz, N., Wainfan, E., Borek, E. \& Waelsch, H. The enzymatic formation of hydroxamic acids from glutamine and asparagine. J. Bio. Chem. 187, 111-125 (1950).

28. von Caemmerer, S. \& Farquhar, G. D. Some relationships between the biochemistry of photosynthesis and the gas exchange of leaves. Planta 153, 376-387 (1981).

29. Ethier, G. \& Livingston, N. On the need to incorporate sensitivity to $\mathrm{CO}_{2}$ transfer conductance into the Farquhar-von Caemmerer-Berry leaf photosynthesis model. Plant Cell. Environ. 27, 137-153 (2004).

30. Ward, D. A. \& Keys, A. J. A comparison between the coupled spectrophotometric and uncoupled radiometric assays for RuBP carboxylase. Photosynth. Res. 22, 167-171 (1989).

31. Scheibe, R., Fickenscher, K. \& Ashton, A. R. Studies on the mechanism of the reductive activation of NADP-malate dehydrogenase by thioredoxin $m$ and low-molecular-weight thiols. Biochim. Biophys. Acta 870, 191-197 (1986).

32. Rao, M. V. \& Ormrod, D. Ozone exposure decreases UVB sensitivity in a UVBsensitive flavonoid mutant of Arabidopsis. Photochem. Photobiol. 61, 71-78 (1995).

33. Nakano, Y. \& Asada, K. Hydrogen peroxide is scavenged by ascorbate-specific peroxidase in spinach chloroplasts. Plant Cell Physiol. 22, 867-880 (1981).

34. Halliwell, B. \& Foyer, C. H. Ascorbic acid, metal ions and the superoxide radical. Biochem. J. 155, 697-700 (1976).

35. Livak, K. J. \& Schmittgen, T. D. Analysis of relative gene expression data using real-time quantitative PCR and the $2^{-\Delta C T}$ method. Methods 25, 402-408 (2001).

36. Wang, Y. et al. Tomato HsfA1a plays a critical role in plant drought tolerance by activating ATG genes and inducing autophagy. Autophagy 11, 2033-2047 (2015).

37. Fracassini, D. S. \& Del Duca, S. Biochemistry and function of plant transglutaminases. Minerva Biotecnol. 14, 135 (2002).

38. Ioannidis, N. E. et al. Remodeling of tobacco thylakoids by over-expression of maize plastidial transglutaminase. Biochim. Biophys. Acta 1787, 1215-1222 (2009).

39. Ioannidis, N. E., Malliarakis, D., Torné, J. M., Santos, M. \& Kotzabasis, K. The overexpression of the plastidial transglutaminase from maize in Arabidopsis increases the activation threshold of photoprotection. Front. Plant Sci. 7, 635 (2016).

40. Yuan, Y. et al. Effects of exogenous putrescine on chlorophyll fluorescence imaging and heat dissipation capacity in cucumber (Cucumis sativus L.) under salt stress. J. Plant Growth Regul. 33, 798-808 (2014).

41. Del Duca, S., Tidu, V., Bassi, R., Esposito, C. \& Serafmi-Fracassini, D. Identification of chlorophyll-a/b proteins as substrates of transglutaminase activity in isolated chloroplasts of Helianthus tuberosus L. Planta 193, 283-289 (1994).

42. Ortigosa, S. M. et al. Oxidative stress induced in tobacco leaves by chloroplast over-expression of maize plastidial transglutaminase. Planta 232, 593-605 (2010).

43. Suzuki, Y. \& Makino, A. Translational downregulation of RBCL is operative in the coordinated expression of Rubisco genes in senescent leaves in rice. J. Exp. Bot. 64, 1145-1152 (2013). 
44. Suzuki, Y., Miyamoto, T., Yoshizawa, R., Mae, T. \& Makino, A. Rubisco content and photosynthesis of leaves at different positions in transgenic rice with an overexpression of RBCS. Plant Cell Environ. 32, 417-427 (2009).

45. Cohen, I., Sapir, Y. \& Shapira, M. A conserved mechanism controls translation of Rubisco large subunit in different photosynthetic organisms. Plant Physiol. 141, 1089-1097 (2006)

46. Long, S. P., ZHU, X. G., Naidu, S. L. \& Ort, D. R. Can improvement in photosynthesis increase crop yields? Plant Cell Environ. 29, 315-330 (2006)

47. Cheng, F. et al. Chloroplastic thioredoxin-f and thioredoxin-m 1/4 play important roles in brassinosteroids-induced changes in $\mathrm{CO}_{2}$ assimilation and cellular redox homeostasis in tomato. J. Exp. Bot. 65, 4335-4347 (2014).

48. Moreno, J., García-Murria, M. J. \& Marín-Navarro, J. Redox modulation of Rubisco conformation and activity through its cysteine residues. J. Exp. Bot. 59, 1605-1614 (2008).
49. Jiang, Y. P. et al. Cellular glutathione redox homeostasis plays an important role in the brassinosteroid-induced increase in $\mathrm{CO}_{2}$ assimilation in Cucumis sativus. New Phytol. 194, 932-943 (2012).

50. Irihimovitch, V. \& Shapira, M. Glutathione redox potential modulated by reactive oxygen species regulates translation of Rubisco large subunit in the chloroplast. J. Biol. Chem. 275, 16289-16295 (2000)

51. Gong, B. et al. Overexpression of S-adenosy/methionine synthetase 1 enhances tomato callus tolerance to alkali stress through polyamine and hydrogen peroxide cross-linked networks. Plant Cell Tissue Organ Cult. 124, 377-391 (2016).

52. Foyer, C. H. \& Noctor, G. Ascorbate and glutathione: the heart of the redox hub. Plant Physiol. 155, 2-18 (2011).

53. Foyer, C. H., Lopez-Delgado, H., Dat, J. F. \& Scott, I. M. Hydrogen peroxide-and glutathione-associated mechanisms of acclimatory stress tolerance and signalling. Physiol. Plant. 100, 241-254 (1997). 